\section{Effect of particle size and Debye length on order parameters of colloidal silica suspensions under confinement $\dagger$}

\author{
Yan Zeng, ${ }^{a}$ Stefan Grandner, ${ }^{b}$ Cristiano L. P. Oliveira, $\dagger_{+}^{c}$ Andreas F. Thünemann, ${ }^{d}$ Oskar Paris, ${ }^{e}$ \\ Jan S. Pedersen, ${ }^{c}$ Sabine H. L. Klapp ${ }^{b}$ and Regine von Klitzing ${ }^{* a}$
}

Received 25th May 2011, Accepted 24th August 2011

DOI: 10.1039/c1sm05971h

\begin{abstract}
Using atomic force microscopy (AFM) and small angle X-ray scattering (SAXS), we show a full comparison between structuring of nanoparticles in confinement and in bulk in order to explain the effect of confinement on characteristic lengths and the scaling law of the characteristic lengths. Three different-sized particle suspensions are used to check the generalization and the correlation between the characteristic lengths and the system parameters, like particle diameter and Debye length. The two characteristic lengths obtained from AFM force curves, the oscillatory wavelength $\lambda$, which is related to the average particle distance, and the decay length $\xi$, which measures how far particle correlates to obtain periodic oscillations, are in good agreement with the mean particle distance $2 \pi / q_{\max }$ and the correlation length $2 / \Delta q$ in bulk, respectively, obtained from the structure peaks of SAXS diagrams. Although confinement causes layering of nanoparticles parallel to the confining surfaces, the characteristic lengths in the direction perpendicular to the confining surfaces follow the bulk behavior. The wavelength scales as $\rho^{-1 / 3}$ with the particle number density $\rho$ irrespective of the particle size and the ionic strength and shows a pure volume effect. Upon comparing with literature results, the $\lambda=\rho^{-1 / 3}$ scaling law can be applied more generally for charged particles, as long as the repulsive interaction is sufficiently long-ranged, than the previous expression of $\lambda=2\left(\mathrm{R}+\kappa^{-1}\right)$, which only approaches the value of average particle distance under specific conditions. The decay length $\xi$ is controlled both by the particle size and the ionic strength of the suspensions, and $\xi=\mathrm{R}+\kappa^{-1}$ is proposed in the paper. In addition, the interaction strength, the force amplitude and maximum scattering intensity, increases linearly with particle concentration. On the other hand, the Monte Carlo (MC) simulations and approximate hypernetted chain (HNC) closure calculation based on Derjaguin-Landau-VerweyOverbeek (DLVO) potential are employed to study the characteristic lengths from the theoretical point of view. The experimental wavelengths are in good agreement with the theoretical counterparts and the experimental decay lengths show the same qualitative behavior as theoretical ones on the particle size and ionic strength.
\end{abstract}

${ }^{a}$ Stranski-Laboratorium, Institute of Chemistry, Technical University Berlin, 10623 Berlin, Germany. E-mail: klitzing@mailbox.tu-berlin.de; Fax: +49-(0)30-314-26602; Tel: +49-(0)30-314-23476

${ }^{b}$ Institute of Theoretical Physics, Technical University Berlin, Sec. EW 7-1, 10623 Berlin, Germany

${ }^{c}$ Department of Chemistry, University of Aarhus, Langelandsgade 140, DK-8000 Aarhus C, Denmark

${ }^{d}$ Department of Analytic Chemistry, BAM Federal Institute for Materials Research and Testing, Richard-Willstätter-Straße 11, D-12489 Berlin, Germany

${ }^{e}$ Institute of Physics, Montanuniversitaet Leoben, Franz-Josef Straße 18, A-8700 Leoben, Austria

$\dagger$ Electronic supplementary information (ESI) available. See DOI: $10.1039 / \mathrm{c} 1 \mathrm{sm} 05971 \mathrm{~h}$

† Present address: Instituto de Física, Universidade de São Paulo, Caixa Postal 66318, 05314-970 São Paulo, Brasil

\section{Introduction}

Colloidal suspensions containing nanoparticles have attracted scientists and industries for several decades because of their important applications. For instance, they are widely used in ultra-polishing of electronic materials, as inks and paint pigments in printing, as cosmetics, lotions, pharmaceuticals, ceramics, foams, and emulsions. Thus, the understanding of the interparticle surface force is key for manipulating the stability, rheology and other desired properties of the suspensions. Due to an enhanced interest in miniaturization, the behavior of colloidal nanoparticles in thin films draws increasing research attention.

In the bulk, the surface forces between nanoparticles follow classic Derjaguin-Landau-Verwey-Overbeek (DLVO) theory, resulting from the van der Waals force and electrostatic force. The structuring of nanoparticles in bulk is dependent on the 
particle concentration. The theoretical mean particle distance scales with the particle concentration as $\lambda \propto c^{-1 / 3}$.

When particles are compressed between two surfaces, the particle density profile in the confined region changes with the distance between particle and the confining surface, leading to oscillations with confining surface separation. This confinementinduced oscillation can be measured as oscillatory force, or structure force, ${ }^{1,2}$ and results from the periodic layering of particles parallel to the confining surfaces. The oscillatory wavelength indicates the average particle distance between two adjacent layers of high particle density formed parallel to the confining surfaces, while the decay length is a measure of how far particles correlate to obtain periodic oscillations.

The first study of ordering of nanoparticles can be traced back to the 1980s. Nikolov found that thinning films of aqueous dispersions of polystyrene latex nanoparticles changed thickness with regular step-wise jump transitions by using reflected light microinterferometry. ${ }^{3}$ These observations verified that the stratification of thin liquid films could be explained as a layer-bylayer thinning of ordered structuring of colloidal particles formed inside the film. There are several papers that have also shown that particles tend to form periodic ordering during the approach of confining surfaces by methods of thin film pressure balance, ${ }^{4-6}$ and total reflectometry. ${ }^{7,8}$

Recently, the direct oscillatory force of nanoparticles was obtained by Piech, Tulpar, and Drelich et al. ${ }^{9-11}$ with colloidal probe atomic force microscopy (CP-AFM), which was developed by Ducker and Butt ${ }^{12,13}$ and showed an advantage in measuring the complete oscillatory force curves for various systems. ${ }^{9,10,14-25}$ The oscillatory wavelength of nanoparticles was found to depend on the bulk particle number density $\rho$ according to $\lambda \propto \rho^{-1 / 3}$.

Numerical simulations and density-functional modeling of oscillatory forces on particles entrapped between two planar surfaces were also available. ${ }^{26-29}$ Klapp et al. directly compared the structural forces in a CP-AFM experiment for an aqueous suspension of $26 \mathrm{~nm}$ diameter silica particles using MC simulations of confinement. A good agreement between simulations and experimental results was achieved: the $\lambda \propto c^{-1 / 3}$ scaling was observed.

Previous work, including our last three publications, ${ }^{28-30}$ has experimentally focused on analyzing one key quantity characterizing the oscillatory force: the wavelength. A precise understanding of confinement effect on the full structuring of nanoparticles, namely the oscillatory wavelength and decay length in relation to the bulk counterparts and their relation with the particle size and Debye-Hückel length, is however still missing. Therefore, to achieve better comparison between the bulk solution and the confined film, it is necessary to combine CP-AFM and small angle scattering (SAS) and perform a detailed analysis.

In this paper, AFM, SAXS, and theoretical modelings are combined to study the interaction in suspensions of charged silica nanoparticles. A full comparison of the structuring of particles in confinement and bulk is presented herein, in order to explain the effect of confinement on characteristic lengths and the scaling law of the characteristic lengths. Two characteristic lengths of the suspension as obtained from SAXS are compared to those found under confinement as obtained from AFM results. Both experimental results are compared to the modeling results using DLVO potentials. In addition, we address the effect of the particle size on the interaction strength, i.e. force amplitude from AFM measurements and maximum scattering intensity from SAXS. Instead of using single sized particles, three different silica colloidal nanoparticles with particle diameters of $11 \mathrm{~nm}, 16 \mathrm{~nm}$ and $26 \mathrm{~nm}$ are used for the experiments to check the generalization and the correlation between the characteristic quantities (i.e. wavelength, correlation length, and interaction strength) and the system parameters, like particle diameter and Debye length.

\section{Experimental}

\subsection{Materials}

Three types of Ludox grade colloidal silica nanoparticle suspensions (TMA $34 \mathrm{wt} \%$, HS $40 \mathrm{wt} \%$ and SM $30 \mathrm{wt} \%$, deionized, Aldrich, Germany) were used in AFM force measurements. The original stock of colloidal suspensions were dialyzed with Milli-Q water (Millipore, billerica, MA) for two weeks. The dialysis tubes (Aldrich, Germany) with MWCO of 1000 were used to remove any remaining ions and ionic contaminants. After dialysis, particle suspensions of varying concentrations were prepared with Milli-Q water as solvent. The weight percentage of the solutions were determined by weighing the sample before and after drying $\left(24 \mathrm{~h}\right.$ at $\left.400{ }^{\circ} \mathrm{C}\right)$. The particle size was determined by scanning electron microscopy (SEM S-4000, Hitachi, Japan) at a primary electron energy of $20 \mathrm{keV}$ and by small angle X-ray scattering (see Methods). The $\zeta$-potential was determined by electrokinetic measurements (Zeta Sizer, Malven) at the same conditions employed in the AFM experiments. The corresponding particle diameters, size polydispersity, zeta potentials, and total surface charge $Z$ (see Theoretical Modelings) are summarized in Table 1.

\subsection{Methods}

Colloidal probe AFM. A silica particle (Bangslabs) with radius of $R=3.35 \mu \mathrm{m}$ was glued with epoxy glue (UHU Endfest Plus $300)$ to the end of a tipless rectangular cantilever (CSC12, MikroMasch, Estonia) using a three-dimensional microtranslation stage according to the previously reported procedure. ${ }^{12}$ Immediately before each experiment the silica sphere with cantilever was cleaned by exposure to a plasma cleaner for $20 \mathrm{~min}$ to remove all the organic contaminants and to create a high density of hydrophilic silanol groups $(\mathrm{Si}-\mathrm{OH})$ on the surface. The substrate was a silicon wafer, cleaned with RCA method ${ }^{31}$ and then thoroughly rinsed with Milli-Q water. The cantilever was placed into a cantilever holder and the probe was positioned roughly a few $\mu \mathrm{m}$ above the nitrogen stream dried silicon wafer

Table 1 Experimental results for the diameters $\sigma$, size polydispersity $s$, $\zeta$ potentials, and total surface charge $Z$ of the Ludox particles investigated in the study

\begin{tabular}{llllr}
\hline Type & $\sigma / \mathrm{nm}$ & $s$ & $\zeta / \mathrm{mV}$ & $Z$ \\
\hline TMA 34 & $26 \pm 2$ & 0.12 & -60 & 35 \\
HS 40 & $16 \pm 2$ & 0.14 & -57 & 13 \\
SM 30 & $11 \pm 2$ & 0.19 & -56 & 6 \\
\hline
\end{tabular}


surface. Deflection vs Zsensor curves were measured with a commercial atomic force microscope MFP produced by Asylum Research, Inc. and distributed by Atomic Force (Mannheim, Germany). With this instrument the position of the cantilever was controlled and determined by $z$-axis position sensor equipped with the piezoelectric translator and the cantilever deflection was determined from the motion of the laserbeam reflected from the back of the microfabricated silicon cantilever. Each cantilever spring constant was individually determined by thermal noise power spectra before or after the experiment, ${ }^{32}$ which yielded values in the range $0.01-0.08 \mathrm{~N} \mathrm{~m}^{-1}$.

The scanning frequencies were varied from 0.5 to $1 \mathrm{~Hz}$ over a scan size of $300-400 \mathrm{~nm}$, which corresponded to approach velocities in the range of $150-400 \mathrm{~nm} \mathrm{~s}^{-1}$. Chan and Engel showed that hydrodynamic drainage forces were negligible at these approach speeds. ${ }^{33,34}$ For each sample solution, altogether $30-40$ force-distance curves were recorded at the same lateral position of the silicon wafer.

To quantitatively study the structuring of particle suspensions, the oscillatory forces are fitted with

$$
\frac{F(h)}{R}=A \exp \left(-\frac{h}{\xi}\right) \cos \left(2 \pi \frac{h}{\lambda}-\theta\right)+\text { offset },
$$

where $R$ is the radius of the colloidal probe, $\lambda$ is related to the length of the average particle interlayer distance, $\xi$ is how far particle ordering correlates to obtain periodic oscillations, $A$ the strength of the particle interaction, $\theta$ the phase shift, and offset the force offset. Force per cantilever radius $\frac{F(x)}{R}$ is the measure of interaction energy per area. The silica sphere is $6.7 \mu \mathrm{m}$ in diameter, thus by Derjaguin approximation the sphere surface can be considered as flat surface because of the comparatively small force distance (normally $<300 \mathrm{~nm}$ ). In the present work, we focus on the three important parameters characterizing the oscillations: $\lambda, \xi$ and $A$.

SAXS. The SAXS measurements were performed on small angle X-ray equipment SAXSess $\mathrm{mc}^{2}$ (Anton Paar, Graz). The equipment used a sealed tube to generate $\mathrm{X}$-ray $(\mathrm{Cu} \mathrm{K} \alpha$, $0.1542 \mathrm{~nm}$ ) and consisted of a line collimation system. Sampledetector distance was $309 \mathrm{~mm}$. A fluid flow cell with $1 \mathrm{~mm}$ quartz capillary was used. For each sample, the output intensity was the integral of 100 frames of measurements. The data were first normalized using the primary beam intensity as a standard. The water background was subtracted and then the desmearing process was performed using corresponding beam length and width profiles. At the end, the structure factor was extracted by dividing the form factor $F(q)$ from the total intensity. The structure peak which indicates interparticle interactions within the suspensions has a Lorentzian line shape, produced from a Fourier transformation on an exponential function of $g(\mathrm{r}) \propto \exp \left(-r /\left(\frac{2}{\Delta q}\right)\right) \cos \left(q_{\max } r\right) . .^{35,36}$ The structure peak is fitted with

$$
S(q)=\frac{S_{0}\left(\frac{\Delta q}{2}\right)^{2}}{\left(q-q_{\max }\right)^{2}+\left(\frac{\Delta q}{2}\right)^{2}}+y_{0}
$$

where $S_{0}$ denotes the structure peak intensity, $\Delta q$ the full width at half maximum of the intensity, $q_{\max }$ the center, and $y_{0}$ the baseline of the peak.

\section{Theoretical modelings}

Based on DLVO theory the charged suspension is modeled on an effective level which only includes the negatively charged silica particles explicitly. ${ }^{27-29,37}$ Here, we use the electrostatic part of the DLVO potential ${ }^{38}$

$$
u(r)=\tilde{Z}^{2} e_{0}^{2} \frac{\exp (-\kappa r)}{4 \pi \varepsilon_{0} \varepsilon r},
$$

where $e_{0}$ is the elementary charge, $\varepsilon_{0}$ and $\varepsilon$ are the permittivity of the vacuum and the solvent dielectric constant, respectively, $Z=$ $4 \pi(\sigma / 2)^{2} q_{\mathrm{s}}$ is the total charge of a particle with diameter $\sigma$ and surface charge density $q_{\mathrm{s}}$, and $\tilde{Z}=Z \exp (\kappa \sigma / 2) /(1+\kappa \sigma / 2)$ is an effective valency involving the inverse Debye screening length $\kappa$, which is defined as

$$
\kappa=\left(e_{0}^{2} / \varepsilon_{0} \varepsilon k_{\mathrm{B}} T\right)^{1 / 2}\left(Z \rho+2 I_{\text {salt }} N_{\mathrm{A}}\right)^{1 / 2},
$$

where $\rho$ is the particle number density, $k_{\mathrm{B}}$ is Boltzmann's constant, $T$ is the temperature (set to $300 \mathrm{Kelvin}$ ), and $I_{\text {salt }}$ is the ionic strength of the added salt, where $10^{-5} \mathrm{~mol} \mathrm{~L}^{-1}$ and $10^{-4} \mathrm{~mol}$ $\mathrm{L}^{-1}$ have been used.

For numerical reasons the repulsive part of a Lennard-Jones potential is added to the particle interaction which is, however, small compared to the DLVO interaction energies at typical mean particle distances in this study.

Corresponding to the experiments, the simulations are carried out with $26 \mathrm{~nm}$ and $16 \mathrm{~nm}$ sized particles at room temperature. Following our previous studies, their surface charges have been determined using the Grahame equation ${ }^{39}$ also taking into account the slightly differing $\zeta$-potentials. This yields charges of $Z=35$ for the large particles and $Z=13$ for the smaller ones. These parameters are summarized in Table 1 . The smallest particles with $\sigma=11 \mathrm{~nm}$ and low charges of $Z=6$ are not considered here since the amplitudes of the simulated oscillatory surface forces are too small to extract information at the densities of interest.

Characteristic lengths as wavelength and decay length of bulk systems are extracted from bulk correlation functions $g_{\mathrm{b}}(r)$. Within MC simulations, $g_{\mathrm{b}}(r)$ is determined using $N=500-2000$ particles. Additionally, the exact Ornstein-Zernike integral equation combined with the approximate hypernetted chain (HNC) closure was solved by analyzing the complex poles $q=$ $\pm q_{1}+i q_{0}$ of the structure factor $S_{b}(q)=1+\rho \tilde{h}_{\mathrm{b}}(q)$, where $h_{\mathrm{b}}(r)=$ $g_{\mathrm{b}}(r)-1$. This yields an analytical description of the asymptotic behavior of $h_{\mathrm{b}}(r)$ which reads

$$
r h_{\mathrm{b}}(r)=A_{\mathrm{b}} \exp \left(-q_{0} r\right) \cos \left(q_{1} r-\theta_{\mathrm{b}}\right), r \rightarrow \infty
$$

with $q_{0}=\xi_{\mathrm{b}}{ }^{-1}$ playing the role of an inverse correlation length and $q_{1}=2 \pi / \lambda_{\mathrm{b}}$ determining the wavelength of the oscillation.

The confining surfaces are modeled by two infinite plane parallel, smooth, charged surfaces separated by a distance $h$ along the $z$-direction. ${ }^{37}$ Their surface charge mimics the experimental conditions using silica surfaces with a surface potential of 
$-80 \mathrm{mV}$. To this end we use a modified fluid-wall potential developed in our previous study. ${ }^{37}$ It takes additional wall counterions into account which change the screening between charged walls and colloidal particles. This induces a nonmonotonic behavior of the fluid-wall interaction potential as a function of the wall charge. Using this modification we already showed in ref. 37 that the observations are consistent with experimental findings and the asymptotic properties of the solvation forces (or solvation pressures) concerning wavelengths and decay lengths do not change.

In a confined system the solvation pressure, $f(h)=P_{z z}(h)-P_{\mathrm{b}}$, with $P_{\mathrm{b}}$ being the bulk pressure and $P_{z z}(h)$ the normal component of the pressure tensor, ${ }^{40}$ exhibits oscillations with varying surface separation. This quantity is related to the solvation forces of the AFM experiment via Derjaguin's approximation..$^{41}$ The functions $f(h)$ are fitted according to the expression

$$
\tilde{f}(h)=A_{\mathrm{f}} \exp \left(-\frac{h}{\xi_{\mathrm{f}}}\right) \cos \left(\frac{2 \pi h}{\lambda_{\mathrm{f}}}-\theta_{\mathrm{f}}\right),
$$

with $\lambda_{\mathrm{f}}$ and $\xi_{\mathrm{f}}$ being the wave and decay lengths, respectively. As eqn (5) is derived from Ornstein-Zernike theory, eqn (6) and the similar eqn (1) are only valid for $h \rightarrow \infty$. However, our study shows that the fitting procedure works quite well for small distances starting after the first pressure minimum.

Monte Carlo simulations in the grand canonical (GCMC) ensemble have been employed to investigate the confined systems. The inverse Debye screening length $\kappa$ of the fluid-fluid interaction in eqn (3) is set to the corresponding bulk value at the same chemical potential. Note that $\kappa$ does not include wall counterions. However, the screening parameter of the fluid-wall interaction contains both contributions, i.e. the counterions of the colloids and the walls. Both choices are justified in ref. 37.

In parallel, the confining surfaces are also modeled by two uncharged surfaces since the asymptotic behavior of wavelength and decay length is expected not to change with the confining surface charge. ${ }^{37}$ The fluid-wall potential is chosen to be purely repulsive and decays as position $z^{-9} .{ }^{28,29}$

\section{Results and discussions}

Fig. 1 shows some examples of AFM force versus distance curves for three series of Ludox suspensions at varying concentration. All force curves show oscillations with exponential decay. In general, for the samples at a given particle size at higher concentrations, a layered structuring formed until a much larger separation $(150 \mathrm{~nm})$ was reached. As particle concentration was reduced, the interaction between the particles was reduced as well, resulting in fewer layering transitions. The force amplitude was more pronounced at higher particle concentrations while more gradual transitions were observed at lower particle concentrations. The wavelength of oscillation decreased with particle concentration in the meantime, which indicated that the particle interlayer distance became smaller. In addition, the oscillatory forces were steeper for large-sized particles at a fixed concentration. To understand better the structuring of Ludox nanoparticles under slit-pore confinement, fitting was performed to obtain quantitative values of $\lambda, \xi$ and $A$. The fitting curves by eqn (1) are shown in Fig. 1 as solid lines.

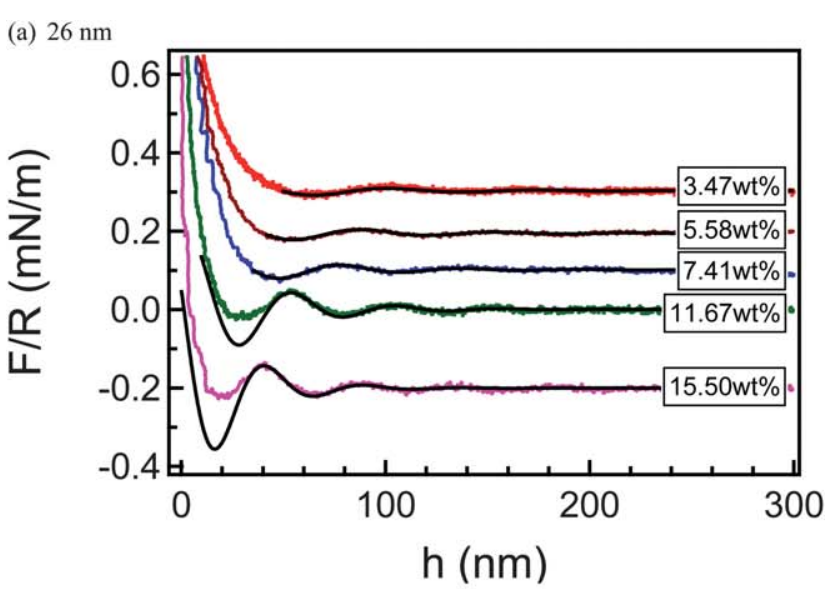

(b) $16 \mathrm{~nm}$

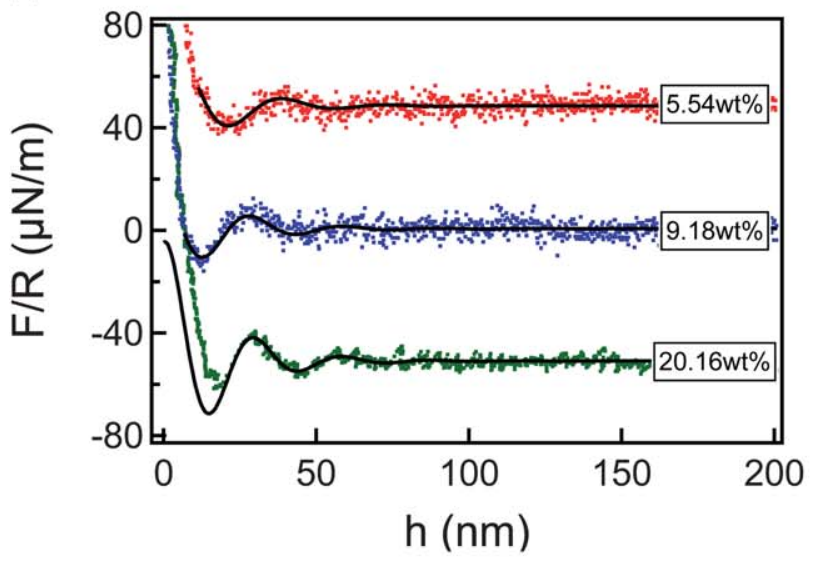

(c) $11 \mathrm{~nm}$

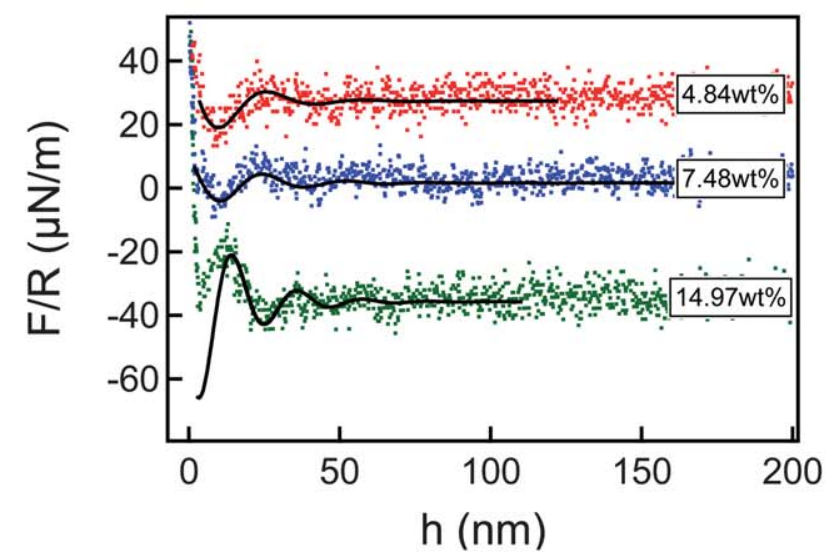

Fig. 1 AFM force curves of three series of Ludox nanoparticle suspensions under slit-pore confinement. For better viewing, the curves are offset vertically. Solid lines are the corresponding curves fitted by eqn (1).

SAXS diagrams are shown in Fig. 2. With increasing sample concentration, the structure peak position shifts to the high $q$ region. Thus a length calculated by $2 \pi / q_{\max }$ decreased with increasing particle concentration. The grey lines in the figure are the corresponding form factors $F(q)$ calculated using the polydisperse sphere model. The polydispersity and sphere size are shown in Table 1. It is apparent that the form factor does not change with concentration, thus the structure factor can be 
(a) $26 \mathrm{~nm}$

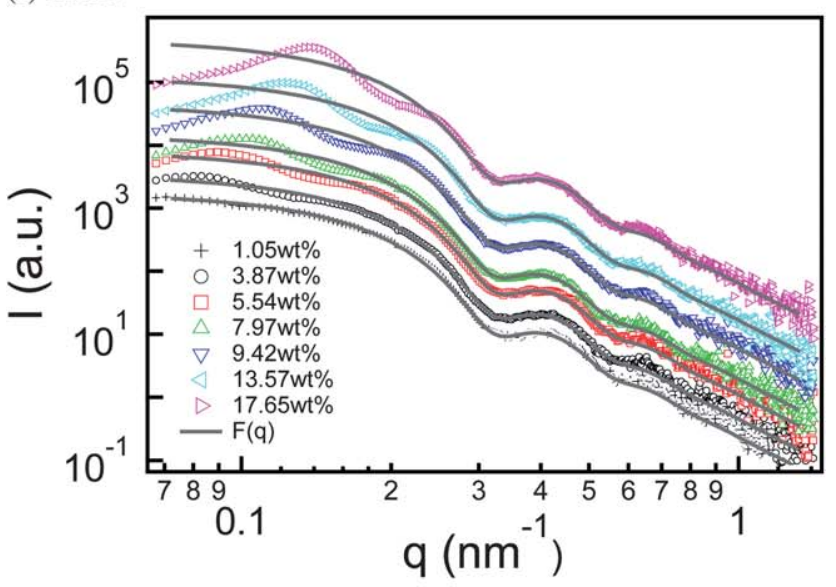

(b) $16 \mathrm{~nm}$

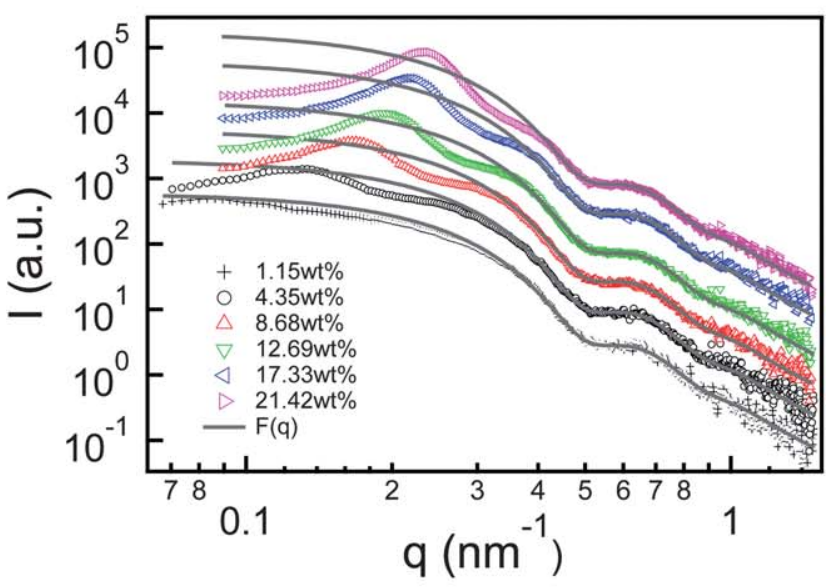

(c) $11 \mathrm{~nm}$

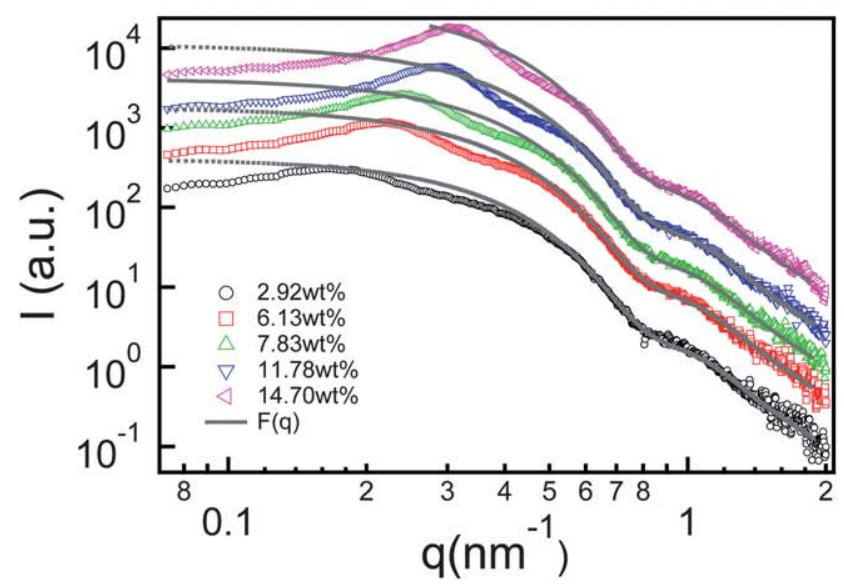

Fig. 2 SAXS diagrams of Ludox nanoparticle suspensions of three different particle sizes at different concentrations. Grey lines represent the best form factor $F(q)$ fitted with polydisperse sphere model.

extracted by dividing the form factor $F(q)$ from the total intensity. Fig. 3 shows the corresponding structure factors for all three series of Ludox samples, including fitted curves with Lorentzian equation (eqn (2)), from which the quantitative values of $q_{\max }$ and $\Delta q$ can be obtained. As particle concentration increases, the position of maximum $q_{\max }$ shifts to higher $q$ values and its width (a) $26 \mathrm{~nm}$

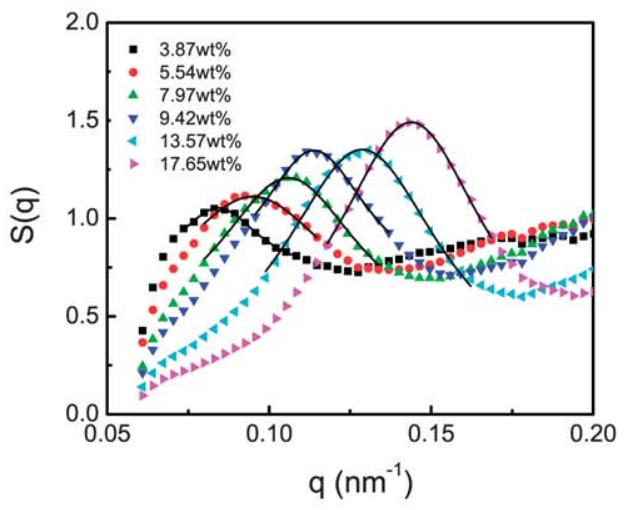

(b) $16 \mathrm{~nm}$

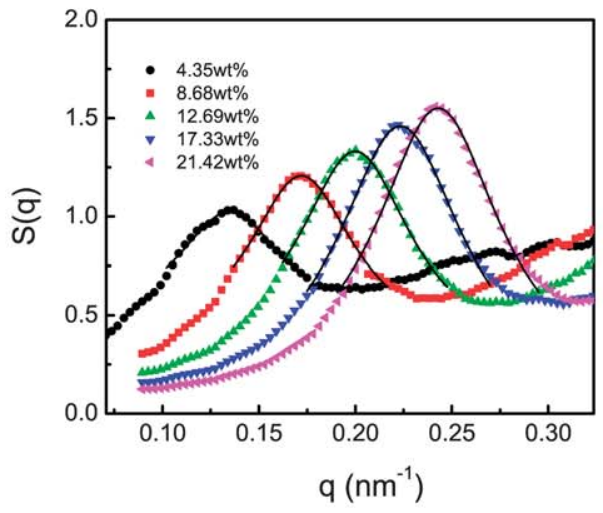

(c) $11 \mathrm{~nm}$

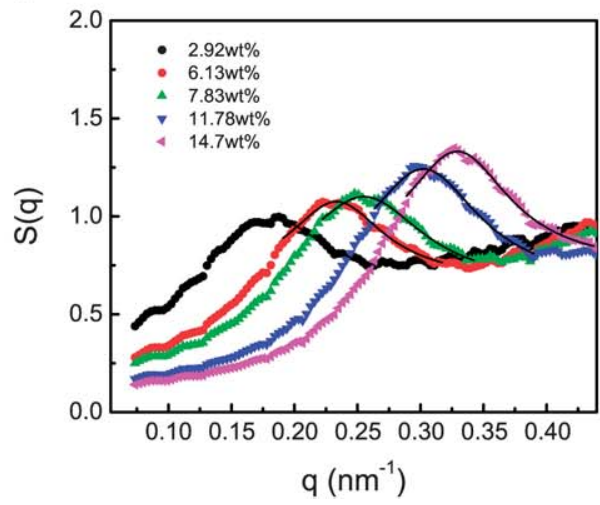

Fig. 3 The structure factors extracted from SAXS intensity. Peaks are fitted to the Lorentzian form of eqn (2) to obtain the quantitative values of $q_{\max }$ and $\Delta q$.

$\Delta q$ increases. Here, $2 / \Delta q$ corresponds to the decay length of pair correlation function $g(r)$, thus it can also be called the correlation length of the particle interaction. This is reminiscent of the decay length of the oscillatory force measured by AFM under slit-pore confinement. Under the assumption that the Bragg relation is valid, the mean particle distance is the reciprocal of the peak position, $2 \pi / q_{\max }$, and can be compared to the wavelength of the oscillation from AFM measurements.

Thus we have two important characteristic lengths from each measurement to analyze and compare: a) mean particle distance in bulk $2 \pi / q_{\max }$ versus wavelength under confinement $\lambda$ and $b$ ) correlation length of the interaction in bulk $2 / \Delta q$ versus the decay 


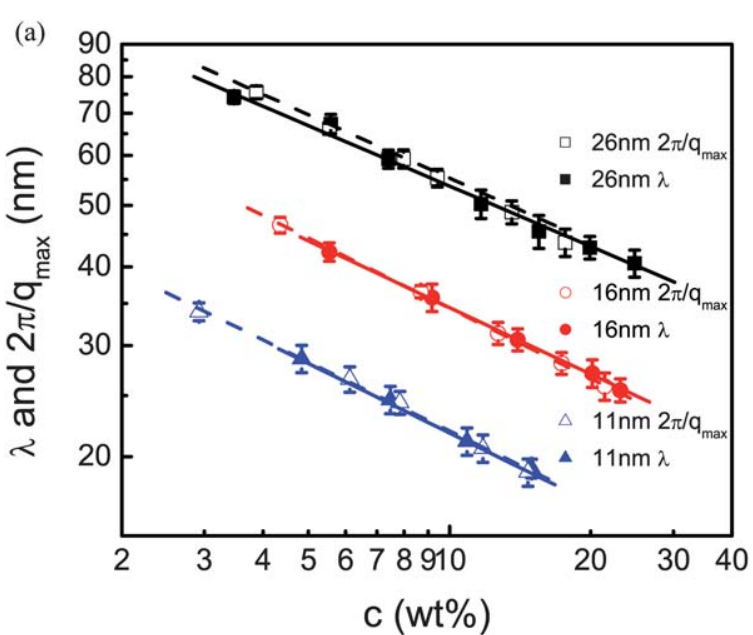

(b)

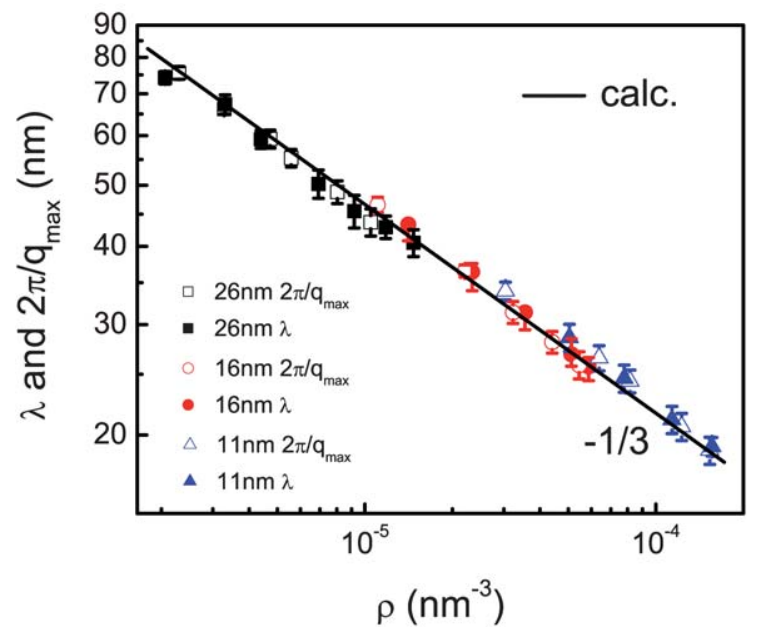

Fig. 4 (a) Comparison between AFM wavelength $\lambda$ and SAXS $2 \pi / q_{\max }$ for three series of particles at varying concentrations. Solid lines are the corresponding fitted curves with scaling factor of approximately -0.33 . (b) The master curve of Fig. 4(a). The solid line is the calculated ideal value of average particle distance in bulk with $\lambda_{\text {ideal }}=\rho^{-1 / 3}$.

length under confinement $\xi$. The better understanding of these characteristic lengths can reveal the effect of confinement on the interaction and then the ordering of the particles. In addition, c) the force amplitude $A$ and maximum scattering intensity $I_{\max }$ can be compared in a qualitative way as well.

\section{a) AFM force wavelength $\lambda$ and SAXS mean particle distance $2 \pi / q_{\max }$}

Fig. 4(a) shows the comparison of $\lambda$ and $2 \pi / q_{\max }$ in a double logarithmic scale. For all three series of Ludox samples, $\lambda$ and $2 \pi / q_{\max }$ scale with particle concentration as an exponent of $-1 / 3$ and both values are in remarkable agreement. $\lambda$ and $2 \pi / q_{\max }$ decrease with increasing particle size at a fixed particle concentration. This is due to the different particle number density at a given weight concentration for particles with unequal size, i.e. using smaller particles lead to a larger number density at the same weight concentration. Thus, the particle concentration needs to be converted into particle number density in order to analyze the effect of size and associated surface charge on the characteristic length. Fig. 4(b) shows the corresponding plot. $\lambda$ and $2 \pi / q_{\max }$ for all three series of samples coincide with the ideal curve calculated from $\lambda_{\text {ideal }}=\rho^{-1 / 3}$, which is the average distance between particles in the bulk. The agreement of both characteristic lengths to the particle-size-independent ideal value indicates that the interparticle distance is solely number density dependent, not influenced by the particle size and associated surface charge, whether in bulk or confinement. This observation is also in agreement with previous work. ${ }^{30}$

Several literature studies have shown that the wavelength (in AFM, or step size in thin film pressure balance) coincided with the effective particle diameter, including the Debye length, for colloidal samples at high concentration (above $20 \mathrm{wt} \%$ )., ${ }^{3,19,21}$ Here, we first compare the AFM wavelength with the calculated effective particle diameter, $2\left(R+\kappa^{-1}\right)$. The $\kappa^{-1}$ calculated from eqn (4) and determined from conductivity measurements have similar values, which are in the range of approximately $0.7-1.5 \sigma$ for the particle concentration of interest (discussed in ESI $\dagger$ ). It is obvious that the AFM wavelength is significantly smaller than the corresponding effective particle diameter in the absence of interactions between particles, as shown in Fig. 5. This indicates that the particles' counterion double layers overlapped significantly in our studied concentration range, leading to the strong electrostatic repulsion. In the meantime, our AFM results showed the $\rho^{-1 / 3}$ scaling law was valid at least until $24.8 \mathrm{wt} \%$ (the maximum experimentally studied concentration corresponds to $13 \mathrm{vol} \%$ ) and GCMC results extended the validity until $30 \mathrm{vol} \%{ }^{29}$ Upon fitting the literature results, which claimed to be close to the effective particle size, the scaling law of $-1 / 3$ was still obtained. ${ }^{3,19}$ Therefore, one can claim that interparticle distance is not ionic strength controlled and the $-1 / 3$ scaling law is a general description for the distance of charged particles in the direction normal to the confining walls, as long as the repulsive interaction is sufficiently long-ranged. It is worth mentioning that this scaling law is no longer valid when the interaction is characterized by the hard core of the particle, where the wavelength is the diameter of particle and not affected by the bulk

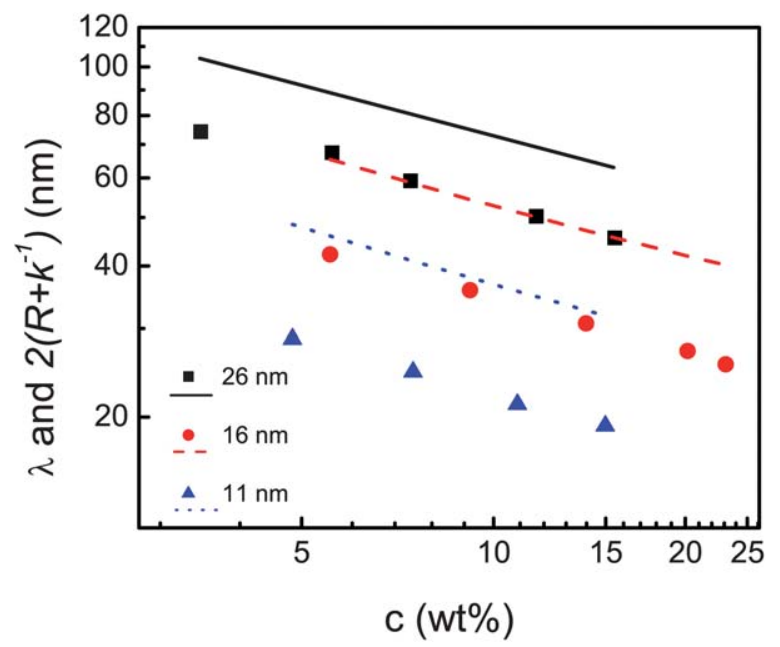

Fig. 5 Comparison between AFM wavelength $\lambda$ and the calculated effective particle diameter, $2\left(R+\kappa^{-1}\right)$. Solid line: $2\left(R+\kappa^{-1}\right)$ for $26 \mathrm{~nm}$, dashed line: $2\left(R+\kappa^{-1}\right)$ for $16 \mathrm{~nm}$, dotted line: $2\left(R+\kappa^{-1}\right)$ for $11 \mathrm{~nm}$. 


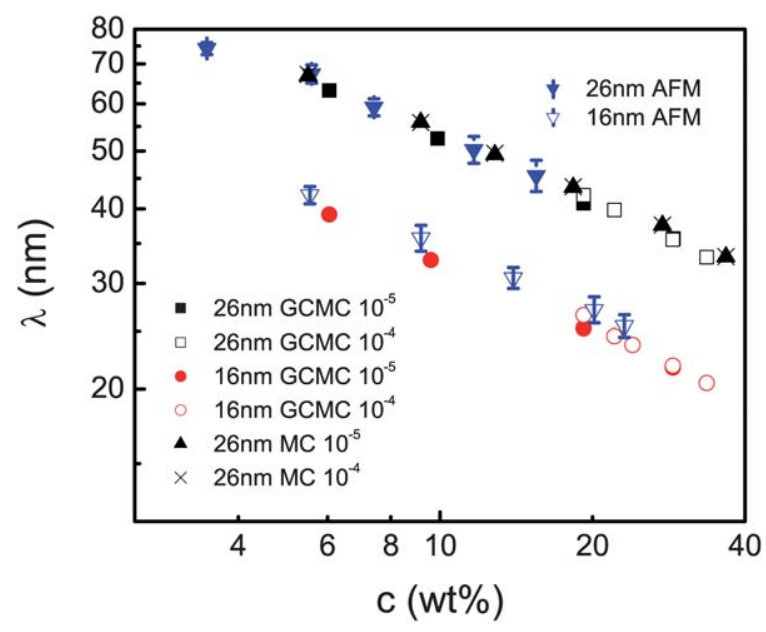

Fig. 6 Asymptotic wavelength as a function of the particle concentration from MC simulation for bulk and GCMC for confinement (between two uncharged walls) at varying extra salt concentration and the wavelength from AFM experiments. The GCMC results from two charged walls have the same values as those from uncharged walls (not shown).

concentration, for example in the case of nonionic surfactant micelles $^{42}$ and charge screened particles at sufficiently high concentration or high ionic strength. The previous description of $2\left(R+k^{-1}\right)$ only in some systems (depends on ionic strength of the samples) approaches the value of wavelength at high concentrations.

Additionally, the theoretical results were obtained with GCMC under slit-pore confinement by modeling between two uncharged walls. Fig. 6 shows the comparison of GCMC asymptotic wavelength with the AFM oscillatory wavelength for two particle sizes. There is a good agreement between the GCMC results with those from the experiments. The wavelengths obtained from GCMC modeled between two charged walls are the same as those from uncharged walls (not shown). The bulk wavelengths for $26 \mathrm{~nm}$ sized particles obtained from MC simulation at two different ionic strength are presented as well. The theoretical bulk wavelengths coincide well with those in confinement, which coincides with the prediction by DFT calculations. ${ }^{26}$ The consistency of theoretical results and the experimental ones indicates that the simple DLVO-based model is valid to apply on such charged nanoparticles, unrelated to the confining surface charge. The unchanged wavelength with different absolute value of added salt concentration $I_{\text {salt }}$ employed in the calculations indicates that the repulsive interaction between particles is long-ranged, thus the wavelength is not ionic strength influenced.

\section{b) AFM force decay length $\xi$ and SAXS correlation length $2 / \Delta q$}

The fitting of decay length of the oscillatory force is more complicated than that of the wavelength, because a jump-into contact always take place at high concentration, i.e. the curves at concentration higher than $10 \mathrm{wt} \%$ in Fig. 1(a), (b) and (c). This jump is normally due to the relatively low spring constant of the cantilever used in the measurement compared to the strong depletion force caused by the exclusion of the last layer of particles, which leads to mechanical instability in those regions of

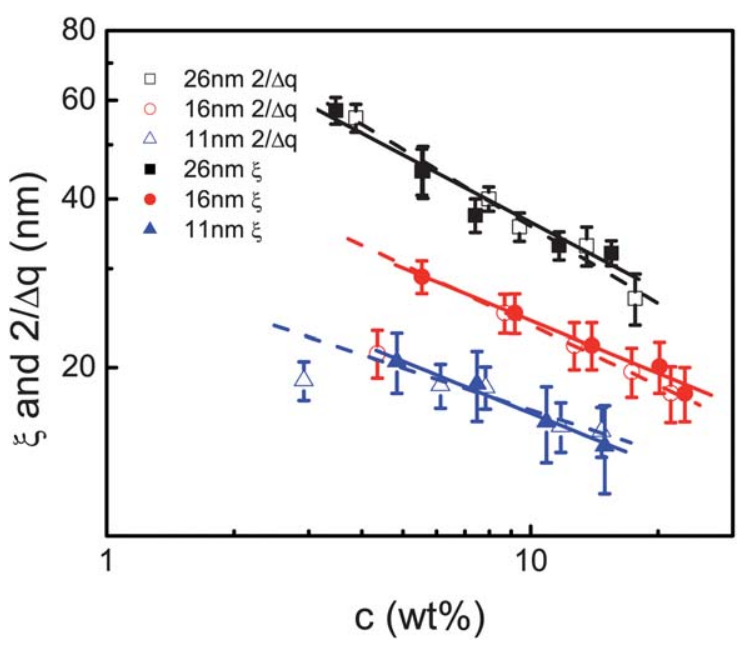

Fig. 7 Comparison between AFM decay length $\xi$ and SAXS correlation length $2 / \Delta q$.

the force curves. The optimal fitting needs to cover the first peak of oscillation instead of the valley for the aforementioned reason.

The comparison between $\xi$ and $2 / \Delta q$ for all three series of samples is shown in Fig. 7. There is a good agreement between $\xi$ and $2 / \Delta q$, except for the initial points of $16 \mathrm{~nm}$ and $11 \mathrm{~nm}$ samples which show deviations from the fit, mainly due to the low resolution of small size particles in the SAXS experiment. Both $\xi$ and $2 / \Delta q$ are measures of the range over which particles in one region are correlated with those in another region. A smaller $\xi$ or $2 / \Delta q$ indicates a smaller interaction distance, which corresponds to a less correlated structure. The oscillation decay length was equal to the range of positional correlations extracted from the SAXS peak width, which suggests that the force decay is indeed mainly caused by the loss of positional correlations.

So far, the agreement between $\xi-2 / \Delta q$ and $\lambda-2 \pi / q_{\max }$ indicates average interparticle distance and the correlation length in the direction perpendicular to the confining surfaces correlated well with the bulk ones. No confinement effect in terms of the average interparticle distance and correlation length was observed at the particle concentration considered. The question

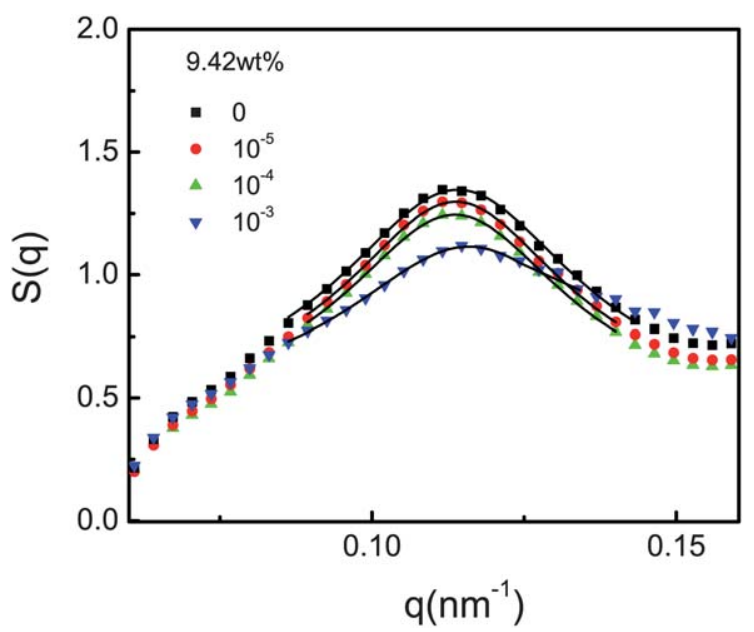

Fig. 8 The structure factor of $26 \mathrm{~nm}$ sized particle suspension with varying salt concentration at a fixed particle concentration. 
then is whether there is an effect of confinement on another scale. The occurrence of an oscillatory force itself is a confinement effect, which is caused by the oscillatory density profiles of particles in confinement and represented as layers of particles with varying particle densities formed parallel to the confining surfaces. This confinement induces layering of nanoparticles, in the vicinity of the confining surfaces, indicating that the translational symmetry of the bulk system is broken. ${ }^{43,44}$ In the particle concentration range considered in this study, the particles within the layers are fluid-like as in the bulk and the asymptotic range is valid until to the first minimum. This fluid-like in-plane structuring was also addressed by previous experimental and theoretical studies at low particle concentrations. $^{29,45-47}$ Those previous studies also showed that a higher ordering started to form within the contact layer as particle concentration increased and the full oscillation deviated from the $\exp () \times \cos ()$ asymptotic behavior. Nevertheless, in the present studied concentration regime, although particles form layering parallel to the confining surfaces, no higher ordering is found within the layers and the asymptotic wavelength and decay length of oscillation in the direction perpendicular to the confining surfaces correlate well with the bulk ones, the wavelength and decay length of the pair correlation function.

The scaling laws in Fig. 7 are $-0.39,-0.33$, and -0.29 for $26 \mathrm{~nm}, 16 \mathrm{~nm}$ and $11 \mathrm{~nm}$ sized particles, respectively. The variance in the scaling law suggests that the particle size has a significant influence on the correlation length. In addition, the structure factor of $26 \mathrm{~nm}$ sized particle at constant particle concentration and varying salt concentration are shown Fig. 8, where the peak broadening and intensity decrease was observed, indicating the correlation length $2 / \Delta q$ decreases with increase in salt concentration. The mean particle distance remained the same up until the point that particles started to form aggregates at concentrations higher than $10^{-3} \mathrm{M}$. The corresponding data $2 / \Delta q$ and $2 \pi / q_{\max }$ are summarized in Table 2. Similar results were obtained in our previous confinement experiment where the correlation length decreased with increasing salt concentration and the wavelength was not affected when $I<10^{-3} \mathrm{M}^{28}$

The correlation length, which indicates the decay length of the ordering, is reminiscent of the Debye screening length. The previous work showed that the decay length of the interaction between two flat surfaces was found as the Debye-Hückel length. ${ }^{48}$ Considering particle geometry, we use $R+\kappa^{-1}$ for the predicted correlation length between particles. Fig. 9 shows the comparison of experimental correlation lengths obtained from AFM force curves and the predicted values by assuming $\xi=$ $R+\kappa^{-1}$. These two values coincided with each other for all sized particles. We further plotted the radius-subtracted correlation

Table 2 SAXS results for the mean particle distance and the correlation length at different concentration of added salt and a fixed particle concentration of $9.42 \mathrm{wt} \%$

\begin{tabular}{lll}
\hline Salt conc. $\left[\mathrm{mol} \mathrm{L}^{-1}\right]$ & $2 / \Delta q / \mathrm{nm}$ & $2 \pi / q_{\max } / \mathrm{nm}$ \\
\hline 0 & 35.7 & 55.2 \\
$10^{-5}$ & 34.8 & 55.3 \\
$10^{-4}$ & 32.7 & 55.4 \\
$10^{-3}$ & 26.5 & 55.1 \\
\hline
\end{tabular}

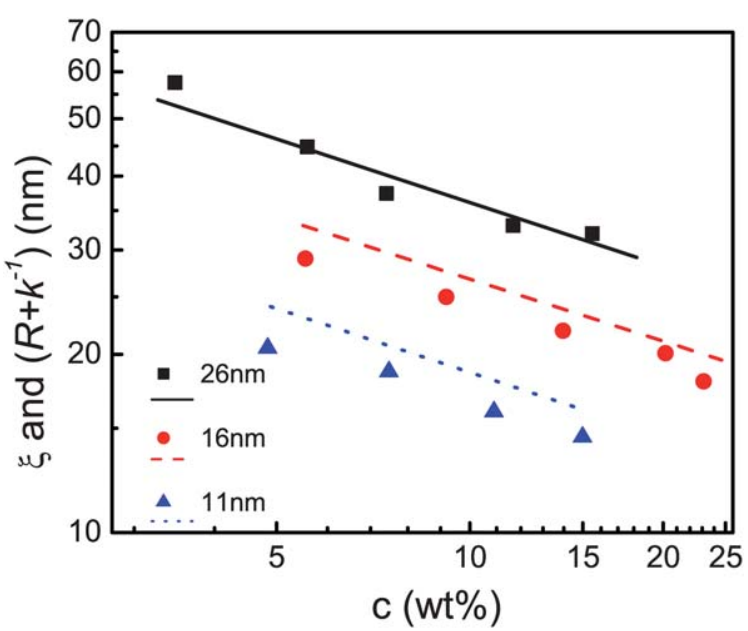

Fig. 9 Comparison between AFM decay length $\xi$ and the proposed correlation length, $R+\kappa^{-1}$. Solid line: $R+\kappa^{-1}$ for $26 \mathrm{~nm}$, dashed line: $R+\kappa^{-1}$ for $16 \mathrm{~nm}$, dotted line: $R+\kappa^{-1}$ for $11 \mathrm{~nm}$.

length versus the total ionic strength of the samples $I_{\text {tot }}$, where $\left(2 N_{\mathrm{A}} I_{\text {tot }}\right)^{-1 / 2}=\left(Z \rho+2 N_{\mathrm{A}} I_{\text {salt }}\right)^{-1 / 2} \propto \kappa^{-1}$. The master curve in fig. 10 shows that for all series of particles, the scaling law of radiussubtracted correlation length with ionic strength is remarkably close to $-1 / 2$. This $-1 / 2$ scaling law with respect to the ionic strength can be suggested to apply to various systems by excluding the geometries of investigated samples. For specific systems, the ionic strength of the solution is attributed solely to the investigated samples (i.e. charged colloids and polyelectrolytes in the absence of added salts). $I_{\text {tot }}$ is then proportional to the sample concentration, thus $-1 / 2$ scaling law of $\xi-R$ with sample concentration $c$ can be applied. ${ }^{24}$ The consistency of measured $\xi$ with $R+\kappa^{-1}$ indicates the correlation length of the present system is both particle size and ionic strength controlled, in contrast to the negligible influence of the particle size and ionic strength on the interparticle distance. The decrease of the correlation length with increase of particle concentration can be understood as a simple screening effect due to increased ionic

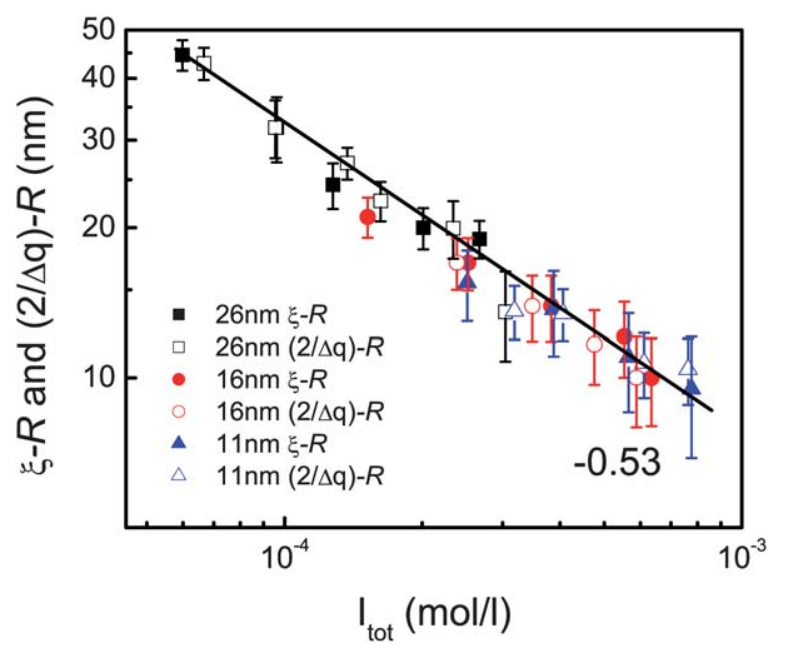

Fig. 10 The radius-subtracted correlation length versus the total ionic strength of the sample. The scaling law for all three series of samples is close to $-1 / 2$, indicating the correlation length is the sum of particle radius and Debye length. 


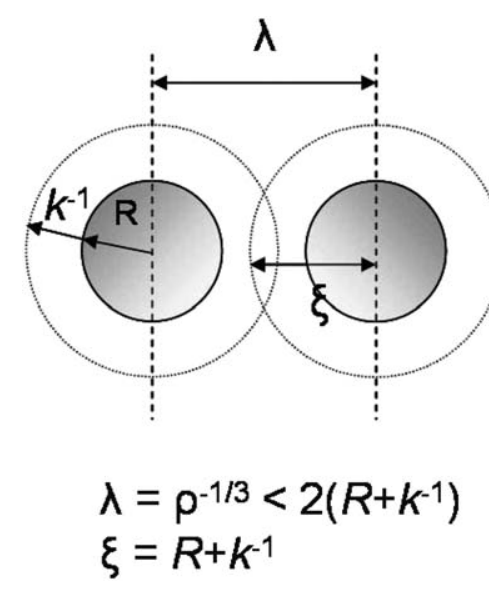

Fig. 11 Schematic representation of the relation between $\lambda, \xi$ and $\kappa^{-1}$ at concentrations considered in this study.

strength associated with particle concentration. The particle concentration affects the correlation length through the ionic strength of the total suspensions instead of through the volume scale for interparticle distance.

The relation of the two characteristic lengths with DebyeHückel length can be schematically represented in Fig. 11. In the present study (low particle concentration regime, salt free), the interparticle distance is always smaller than the effective particle diameter, $\lambda=\rho^{-1 / 3}<2\left(R+\kappa^{-1}\right)$, meaning the diffuse double layers overlap. The correlation length can be proposed as the sum of particle radius and the Debye length, $\xi=R+\kappa^{-1}$.

The theoretical results for the correlation lengths are compared with the experimental results for $26 \mathrm{~nm}$ sized particles at two different ionic strengths $I=10^{-4} \mathrm{~mol} \mathrm{~L}-1$ and $10^{-5} \mathrm{~mol} \mathrm{~L}^{-1}$ plotted in Fig. 12(a). For the bulk system, values of $\xi_{\mathrm{b}}$ were obtained via HNC calculations and MC simulations. As in the SAXS measurements, both theoretical methods exhibit a decrease of the correlation length for increasing particle concentration within the considered parameter range. Although the ionic strength of salt has a significant impact on the correlations, i.e. larger values of $I_{\text {salt }}$ decrease the correlations, it does not change the qualitative behavior described above. However, a quantitative match of the theoretical and experimental data is not observed which might be related to the simple DLVO model used but also differences and inaccuracies in the fitting procedure. The latter is discussed in more detail in the next paragraph. Considering the confined system, similar results regarding the influence of the ionic strength are observed by comparing the AFM measurements with GCMC simulations (using charged and uncharged silica walls) also shown in Fig. 12(a). GCMC simulations modeled between two uncharged silica walls exhibit a clear decrease of the correlation length as AFM results. The simulation results from two charged walls do not exhibit such a clear tendency. Rather, one observes a slight increase of $\xi_{\mathrm{f}}$. However, for larger particle concentrations an increase of $\xi_{\mathrm{f}}$ is indeed expected, ${ }^{28}$ which should not be seen in this parameter range. For GCMC results from charged walls, a larger value of wall surface potential might be set in the simulation than that actually exists in the experiments, which consequently leads to a higher in-plane ordering within the contact layer. Because the determination of $\xi$ in the asymptotic range is quite difficult, the fitting of correlation length might include the data in the nonasymptotic range and thus lead to the deviation from the GCMC results between two uncharged walls. Here we note that, taking the assumed relation $\xi=R+\kappa^{-1}$, the corresponding radiussubtracted decay length versus the ionic strength $I_{\text {tot }}$ obeys a scaling law with exponent $-1 / 2$ for $\mathrm{HNC}$ and GCMC data (uncharged walls) shown in Fig. 12(b). From a theoretical point of view this scaling is explained by the definition of the screening parameter $\kappa^{-1}$ (see eqn (3)).

Whereas the analysis of the simulated correlation functions yielded good values for the wavelength $\lambda_{\mathrm{b}}$ (Fig. 6), the determination of the decay length $\xi_{\mathrm{b}}$ in eqn (5) suffered from big
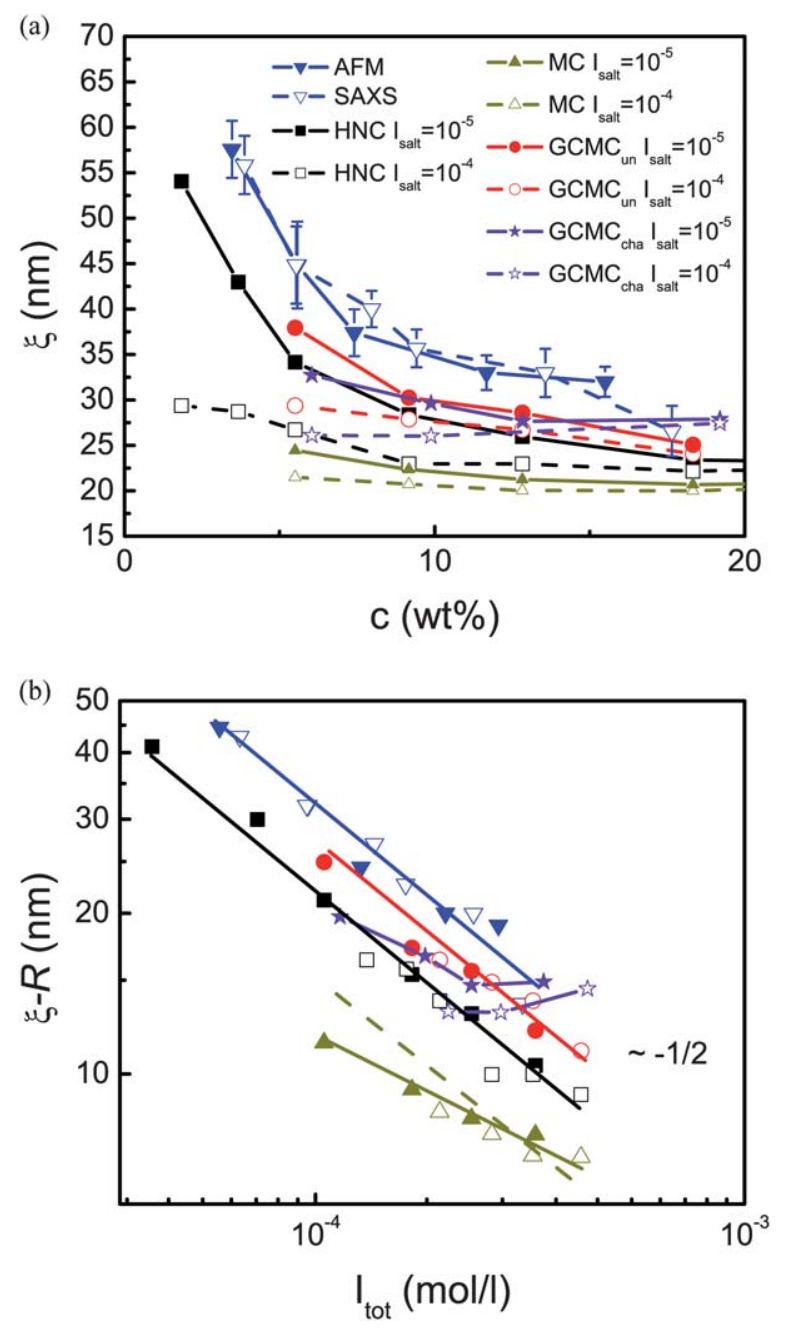

Fig. 12 (a) MC and HNC results for the bulk correlation length as a function of the particle concentration, GCMC for the correlation length in confinement (modeled between two uncharged walls as well as between charged walls) and the experimental results from AFM and SAXS. (b) The radius-subtracted correlation length versus the total ionic strength of the sample. The scaling law of HNC calculation for bulk and GCMC simulation (uncharged walls) for confinement is close to $-1 / 2$, while the scaling law of MC simulation for bulk and GCMC (charged walls) deviates from $-1 / 2$. The difference in the absolute value between experimental and simulated results is due to the uncertainties regarding the asymptotic range that was chosen. 
uncertainties. The fitting procedure of the correlation function using eqn (5) is only valid for the asymptotic range, i.e. for large distances. Choosing a fit range starting after several oscillations should yield better agreement between simulation and theoretical calculation. However, due to statistical errors of the simulations the fitting procedure yielded erroneous results at large $r$ since the amplitudes of $g(r)$ became very small at the bulk concentrations considered. Hence, the limited distance range to fit $g(r)$ yielded uncertainties in $\xi$. As an example of a volume fraction $c=20 \mathrm{wt} \%$ and $I=10^{-5} \mathrm{~mol} \mathrm{~L}^{-1}$ the fit parameter $\xi_{\mathrm{b}}$ with MC model was $\xi_{\mathrm{b}}=20.7 \mathrm{~nm}$ if the fit started after the first peak. Starting after the second peak it was $\xi_{\mathrm{b}}=22.1 \mathrm{~nm}$. Here, we began the fitting after the first peak of $g(r)$ which yielded better agreement with $\mathrm{HNC}$ results than for other fitting ranges used in the previous paper. ${ }^{28}$ However, the qualitative behavior of $\xi_{\mathrm{b}}$, depending on the ionic strength $I$ and the particle concentration, was not affected by the fitting range. The same uncertainty was found using eqn (6) for the correlation length under confinement $\xi_{\mathrm{f}}$, whereas the wavelength $\lambda_{\mathrm{f}}$ yielded good agreement with the experimental results (see Fig. 6). Although HNC directly calculated the correlation functions from integral equations it
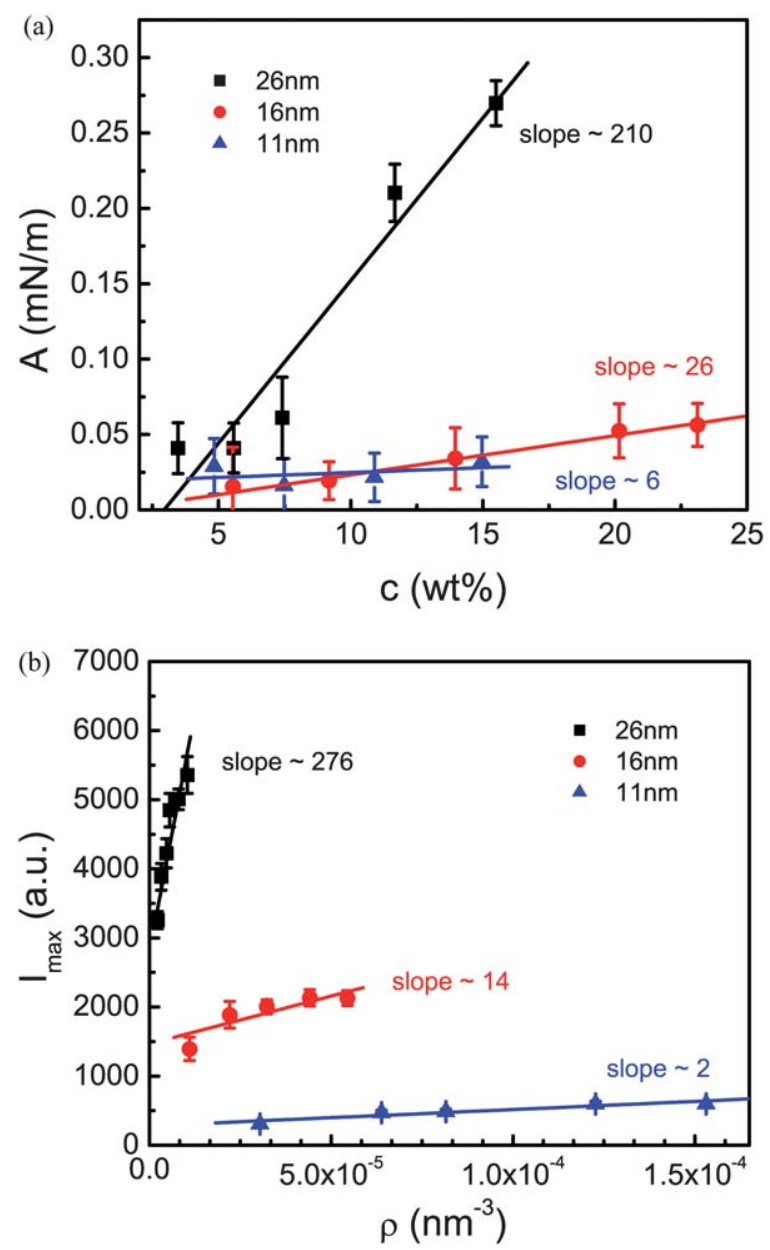

Fig. 13 (a) AFM force amplitude versus particle concentration. Slope ratio $210: 26: 6$ is similar to the ratio of the square of each surface charge $35^{2}: 13^{2}: 6^{2}$. (b) SAXS maximal intensity versus particle number density. Slope ratio $276: 14: 2$ is similar to the square of the particles' volumes, $26^{6}: 16^{6}: 11^{6}$ contained several approximations which might cause the differences to the experimental results as well. As predicted by DFT calculations ${ }^{26}$ the wavelengths $\lambda_{\mathrm{b}}$ and $\lambda_{\mathrm{f}}$ and the correlation lengths $\xi_{\mathrm{b}}$ and $\xi_{\mathrm{f}}$ should be equal, respectively, which was found for the wavelengths, and $\xi_{\mathrm{b}}$ and $\xi_{\mathrm{f}}$ were qualitatively equal to each other which might result from aforementioned reasons.

\section{c) AFM force amplitude and SAXS maximum intensity}

Both AFM force amplitude and SAXS maximum intensity measure the strength of the interactions between particles. As shown in Fig. 13(a) and Fig. 13(b), the force amplitude and maximum intensity increases linearly with particle concentration at fixed size. Analogous behavior was also found for confined polyelectrolyte solutions where the amplitude increased with increasing concentration and polymer charge density due to the stronger increase of all charges. ${ }^{24}$ For larger-sized particles, the increase of amplitude is more pronounced. The ratio of the slopes of the force amplitude curves in Fig. 13(a) equals the ratio of the square of the total charge of particles, $Z^{2}(Z=35,13$, and 6 for $26 \mathrm{~nm}, 16 \mathrm{~nm}$ and $11 \mathrm{~nm}$ sized particles, respectively), indicating that the interaction between particles is electrostatic repulsion dominated and particle surface charge influenced (eqn (3)). Previously, we determined that the increase in particle concentration led to an increase in amplitude while the associated increase in counterion concentration led to a decrease in amplitude. ${ }^{28}$ The SAXS measurements also showed that, at a given particle concentration, increasing salt concentration caused a reduced intensity (Fig. 8). However, the linearly increasing amplitude with no observed maximum indicates that the effect of particle charge dominates the counterion effect in this study. The ratio of the slopes of the maximum scattering intensity versus particle number density in Fig. 13(b) equals the ratio of the particle size with power law of six, $\sigma^{6}$, indicating that scattering intensity is proportional to the product of particle number density and square of the particle volume due to scattering mechanism.

There is no direct way to compare the maximum intensity from SAXS with the force amplitude from AFM in order to know how the interaction strength changes in a confined system. However, the previous work about the influence of confining surface potential on the structuring of particles gave both experimental and theoretical evidence of a pronounced enhancement of force amplitude as the confining surface potential increases. ${ }^{37}$

\section{Conclusions}

The effect of confinement on the ordering of colloidal nanoparticles was studied. Oscillatory force curves obtained by AFM under slit-pore confinement was compared with SAXS results in bulk. Two characteristic lengths describing the structuring of particles were directly compared: oscillatory wavelength $\lambda$ versus mean particle distance $2 \pi / q_{\max }$, and correlation length $\xi$ versus $2 / \Delta q$. In addition, experimental results were compared to the modelings using DLVO potentials.

(1) The good agreement between each characteristic length obtained by AFM and SAXS indicates confinement does not change the average interparticle distance and correlation length of particles in the direction perpendicular to the confining 
surfaces at the concentrations considered, even though the oscillatory force is the consequence of the layering of particles.

(2) The interparticle distance was found neither to be influenced by the particle size nor the ionic strength of the suspensions, but solely dependent on the particle concentration, precisely speaking, the particle number density, with a scaling factor of $-1 / 3$. This implies the interparticle distance is a simple consequence of the volume effect when the repulsive interaction between particle is long-ranged. In addition, a remarkable agreement between experimental interparticle distance and the simulated one was found.

(3) The increase of the particle concentration, the corresponding increase of the total ionic strength, and the decrease of the particle size led to the decrease of the correlation length. The relation between the correlation length and the Debye screening length can be estimated as $\xi=R+\kappa^{-1}$ in the considered concentration regime, meaning $\xi$ is both particle size and ionic strength controlled. This can be motivated by the fact that on one hand in the low particle concentration regime the range of the correlations is determined by the range of the interaction potential. ${ }^{49}$ On the other hand, the range of this potential is determined by the hard-core repulsion with radius $R$ and the DLVO repulsion with range $\kappa^{-1}$ (see eqn (3)). For the bulk system both $\mathrm{HNC}$ and MC simulations provided a qualitative agreement with experimental SAXS results, while in confinement GCMC results between two uncharged walls showed a qualitative agreement with AFM results as well. Hence, the dependence of the correlation length on $R$ and $\kappa^{-1}$ was further proven. GCMC results modeled between two charged walls exhibited a slightly different trend. The differences in the absolute values between theoretical and experimental results and the slightly different behavior for GCMC from two charged walls were due to some uncertainties regarding the asymptotic range and the fit range chosen.

(4) The AFM force amplitude and SAXS maximum intensity both increased linearly with particle concentration. The AFM force amplitude is proportional to the product of particle weight concentration and square of the particle surface charge, indicating that the particle charge exerts a strong effect on the amplitude because the particle-particle interaction is dominated by electrostatic repulsion. The SAXS maximum intensity is proportional to the product of particle number density and the square of particle volume, due to the scattering mechanism.

\section{Acknowledgements}

Y.Z. acknowledges financial support of the Deutsche Forschungsgemeinschaft under SPP 1273 "Kolloidverfahrenstechnik" (KL1165/10-1) and acknowledges TU Berlin. R.v.K. and S.H.K. acknowledge the IGRTG 1524, financed by Deutsche Forschungsgemeinschaft (DFG). Y.Z. and R.v.K. acknowledge Prof. Otto Glatter for the support on SAXS measurements. J.S.P. acknowledges the financial support from The Danish Council for Independent Research Natural Sciences.

\section{References}

1 R. Horn and J. Israelachvili, J. Chem. Phys., 1981, 75, 1400-1411. 2 J. Israelachvili and R. Pashley, Nature, 1983, 306, 249-250.

3 A. Nikolov and D. Wasan, J. Colloid Interface Sci., 1989, 133, 1-12.
4 E. Basheva, K. Danov and P. Kralchevsky, Langmuir, 1997, 13, 4342-4348.

5 N. Denkov, H. Yoshimura, K. Nagayama and T. Kouyama, Phys. Rev. Lett., 1996, 76, 2354-2357.

6 G. Sethumadhavan, A. Nikolov and D. Wasan, J. Colloid Interface Sci., 2001, 240, 105-112.

7 A. Sharma and J. Walz, J. Chem. Soc., Faraday Trans., 1996, 92, 4997-5004.

8 A. Sharma, S. Tan and J. Walz, J. Colloid Interface Sci., 1997, 191, 236-246.

9 M. Piech and J. Walz, J. Phys. Chem. B, 2004, 108, 9177-9188.

10 A. Tulpar, P. Van Tassel and J. Walz, Langmuir, 2006, 22, 2876-2883.

11 J. Drelich, J. Long, Z. Xu, J. Masliyah, J. Nalaskowski, R. Beauchamp and Y. Liu, J. Colloid Interface Sci., 2006, 301, 511-522.

12 W. Ducker, T. Senden and R. Pashley, Nature, 1991, 353, 239-241.

13 H. Butt, Biophys. J., 1991, 60, 1438-1444.

14 A. Milling, J. Phys. Chem., 1996, 100, 8986-8993.

15 A. Milling and K. Kendall, Langmuir, 2000, 16, 5106-5115.

16 S. Biggs, J. Burns, Y. Yan, G. Jameson and P. Jenkins, Langmuir, 2000, 16, 9242-9248.

17 J. Burns, Y. Yan, G. Jameson and S. Biggs, Colloids Surf., A, 2000, 162, 265-277.

18 J. Burns, Y. Yan, G. Jameson and S. Biggs, J. Colloid Interface Sci., 2002, 247, 24-32.

19 A. Nikolov and D. Wasan, Langmuir, 1992, 8, 2985-2994.

20 M. Piech and J. Walz, J. Colloid Interface Sci., 2002, 253, 117-129.

21 C. McNamee, Y. Tsujii, H. Ohshima and M. Matsumoto, Langmuir, 2004, 20, 1953-1962.

22 C. McNamee, Y. Tsujii and M. Matsumoto, Langmuir, 2004, 20, 1791-1798.

23 D. Qu, D. Baigl, C. Williams, H. Mohwald and A. Fery, Macromolecules, 2003, 36, 6878-6883.

24 D. Qu, J. S. Pedersen, S. Garnier, A. Laschewsky, H. Moehwald and R. von Klitzing, Macromolecules, 2006, 39, 7364-7371.

25 Y. Zeng and R. von Klitzing, Soft Matter, 2011, 7, 5329-5338.

26 C. Grodon, M. Dijkstra, R. Evans and R. Roth, Mol. Phys., 2005, 103, 3009-3023.

27 S. H. L. Klapp, D. Qu and R. von Klitzing, J. Phys. Chem. B, 2007, 111, 1296-1303.

28 S. H. L. Klapp, S. Grandner, Y. Zeng and R. von Klitzing, J. Phys.: Condens. Matter, 2008, 20, 494232.

29 S. H. L. Klapp, Y. Zeng, D. Qu and R. von Klitzing, Phys. Rev. Lett., 2008, 100, 118303.

30 S. H. L. Klapp, S. Grandner, Y. Zeng and R. von Klitzing, Soft Matter, 2010, 6, 2330-2336.

31 H. Riegler and M. Engel, Berichte der Bunsen-Gesellschaft-Phys. Chem. Chem. Phys., 1991, 95, 1424-1430.

32 J. Hutter and J. bechhoefer, Rev. Sci. Instrum., 1993, 64, 1868-1873.

33 R. Dagastine, G. Stevens, D. Chan and F. Grieser, J. Colloid Interface Sci., 2004, 273, 339-342.

34 J. Hoh and A. Engel, Langmuir, 1993, 9, 3310-3312.

35 C. Helm, H. Moehwald, K. Kjaer and J. Alsnielsen, Europhys. Lett., 1987, 4, 697-703.

36 A. Spaar and T. Salditt, Biophys. J., 2003, 85, 1576-1584.

37 S. Grandner, Y. Zeng, R. v. Klitzing and S. H. L. Klapp, J. Chem. Phys., 2009, 131, 154702.

38 E. J. W. Verwey and J. T. G. Overbeek, Theory of Stability of Lyophobic Colloids, Elsevier, Amsterdam, 1948.

39 J. N. Israelachvili, Intermolecular and Surface Forces, Academic Press, London, 1992.

$40 \mathrm{M}$. Schoen and S. H. L. Klapp, in Reviews in Computational Chemsitry, Wiley-VCH, New Jersey, 2007, Vol. 24.

41 B. Gotzelmann, R. Evans and S. Dietrich, Phys. Rev. E: Stat. Phys., Plasmas, Fluids, Relat. Interdiscip. Top., 1998, 57, 6785-6800.

42 N. C. Christov, K. D. Danov, Y. Zeng, P. A. Kralchevsky and R. von Klitzing, Langmuir, 2010, 26, 915-923.

43 D. Wasan and A. Nikolov, Nature, 2003, 423, 156-159.

44 M. Chaudhury, Nature, 2003, 423, 131-132.

45 X. Chu, A. Nikolov and D. Wasan, Langmuir, 1994, 10, 4403-4408.

46 D. Wasan, A. Nikolov and B. Moudgil, Powder Technol., 2005, 153, 135-141.

47 S. Grandner and S. H. L. Klapp, J. Chem. Phys., 2008, 129, 244703.

48 I. Sokolov, Q. K. Ong, H. Shodiev, N. Chechik, D. James and M. Oliver, J. Colloid Interface Sci., 2006, 300, 475-481.

49 I. R. Hansen, J. P. McDonald, Theory of Simple Liquids, 3rd edn, Academic Press, Amsterdam, 2006. 